\title{
Discovery of Six New Class II Methanol Maser Transitions, Including the Unambiguous Detection of Three Torsionally Excited Lines toward G 358.931-0.030
}

\author{
S. L. Breen ${ }^{1}$ (D), A. M. Sobolev ${ }^{2}$ (D), J. F. Kaczmarek ${ }^{3}$ (D), S. P. Ellingsen ${ }^{4}$ (D), T. P. McCarthy ${ }^{4}$ (D), and M. A. Voronkov ${ }^{3}$ (D) \\ ${ }^{1}$ Sydney Institute for Astronomy, (SIfA), School of Physics, University of Sydney, NSW 2006, Australia; Shari.Breen@sydney.edu.au \\ ${ }^{2}$ Ural Federal University, 19 Mira street, 620002 Ekaterinburg, Russia \\ ${ }^{3}$ CSIRO Astronomy and Space Science, Australia Telescope National Facility, Box 76, Epping, NSW 1710, Australia \\ ${ }^{4}$ School of Natural Sciences, University of Tasmania, Private Bag 37, Hobart, Tasmania 7001, Australia \\ Received 2019 March 28; revised 2019 April 13; accepted 2019 April 14; published 2019 May 6
}

\begin{abstract}
We present the unambiguous discovery of six new class II methanol maser transitions, three of which are torsionally excited $\left(v_{t}=1\right)$. The newly discovered $6.18 \mathrm{GHz} 17_{-2} \rightarrow 18_{-3} \mathrm{E}\left(v_{t}=1\right), 7.68 \mathrm{GHz} 12_{4} \rightarrow 13_{3} \mathrm{~A}^{-}$ $\left(v_{t}=0\right), 7.83 \mathrm{GHz} 12_{4} \rightarrow 13_{3} \mathrm{~A}^{+}\left(v_{t}=0\right), 20.9 \mathrm{GHz} 10_{1} \rightarrow 11_{2} \mathrm{~A}^{+}\left(v_{t}=1\right), 44.9 \mathrm{GHz} 2_{0} \rightarrow 3_{1} \mathrm{E}\left(v_{t}=1\right)$, and $45.8 \mathrm{GHz} 9_{3} \rightarrow 10_{2} \mathrm{E}\left(v_{t}=0\right)$ methanol masers were detected toward $\mathrm{G} 358.931-0.030$, where the known $6.68 \mathrm{GHz}$ maser has recently been reported to be undergoing a period flaring. The detection of the $v_{t}=1$ torsionally excited lines corroborates one of the missing puzzle pieces in class II maser pumping, but the intensity of the detected emission provides an additional challenge, especially in the case of the very highly excited $6.18 \mathrm{GHz}$ line. Together with the newly detected $v_{t}=0$ lines, these observations provide significant new information that can be utilized to improve class II methanol maser modeling. We additionally present detections of $6.68,19.9,23.1$, and $37.7 \mathrm{GHz}$ class II masers, as well as 36.2 and $44.1 \mathrm{GHz}$ class I methanol masers, and provide upper limits for the 38.3 and $38.5 \mathrm{GHz}$ class II lines. Near simultaneous Australia Telescope Compact Array observations confirm that all 10 of the class II methanol maser detections are co-spatial to $\sim 0.2$ arcsec, which is within the uncertainty of the observations. We find significant levels of linearly polarized emission in the $6.18,6.67,7.68,7.83,20.9,37.7,44.9$, and $45.8 \mathrm{GHz}$ transitions, and low levels of circular polarization in the 6.68, 37.7, and $45.8 \mathrm{GHz}$ transitions.
\end{abstract}

Key words: ISM: individual objects (G358.931-0.030) - ISM: molecules - masers - radio lines: ISM - stars: formation

\section{Introduction}

More than 30 methanol maser transitions have now been detected in the regions surrounding young high-mass stars. These methanol maser transitions have been empirically divided into two classes: class I and class II (e.g., Batrla et al. 1987; Menten 1991), reflecting the respective pumping schemes and therefore their relationship to the exciting object. Class I methanol masers are pumped via collisions (e.g., Leurini et al. 2016; Sobolev \& Parfenov 2018) and so tend to be associated with the shocked gas surrounding outflows and expanding H II regions (e.g., Kurtz et al. 2004; Voronkov et al. 2014). Class II methanol masers are pumped via infrared radiation (e.g., Sobolev et al. 1997a; Cragg et al. 2005) and are exclusively found co-located with young high-mass stars (e.g., Minier et al. 2003; Xu et al. 2008; Breen et al. 2013).

Class II methanol masers at $6.68 \mathrm{GHz}$ have been studied extensively, and a large portion of the Galactic plane has been completely examined as part of the Methanol Multibeam (MMB) Survey (Green et al. 2009) in order to find this transition, resulting in the detection of 972 sources (Breen et al. 2015). Further, targeted searches have discovered a total of 18 class II methanol maser transitions (Ellingsen et al. 2012; Zinchenko et al. 2017, and references therein), some of which are relatively common, such as the $12.2 \mathrm{GHz}$ transition (e.g., associated with $45 \%$ of $6.68 \mathrm{GHz}$ MMB masers; Breen et al. 2016). Other lines with reasonable observed sample sizes (e.g., 19.9, 23.1, 37.7, 38.3, 38.5, 85.5, 86.6, 107.0, and 156.6 GHz; Caswell et al. 2000; Ellingsen et al. 2003, 2004, 2011; Cragg et al. 2004) have revealed lower detection rates, reflecting that the slightly different specific conditions required to produce strong maser emission in these transitions (e.g., Cragg et al. 2005) are less prevalent that those required for the more readily inverted and common 6.68 and $12.2 \mathrm{GHz}$ transitions.

Studies of class I methanol maser emission have been mostly limited to targeted observations in the 36.2, 44.1, 84, and $95 \mathrm{GHz}$ transitions (e.g., Kurtz et al. 2004; Ellingsen 2005; Cyganowski et al. 2009; Chen et al. 2011; Voronkov et al. 2014; Breen et al. 2019), but some complete searches for the 36.2 and $44.1 \mathrm{GHz}$ transitions exist, revealing large numbers of maser sources (e.g., Yusef-Zadeh et al. 2013; Jordan et al. 2015, 2017).

Current class II methanol maser pumping theories are able to broadly account for the observed methanol maser transitions and properties detected in prominent maser sources (e.g., Cragg et al. 2005). In order to reproduce the observed brightness of the common and strong 6.68 and $12.2 \mathrm{GHz}$ class II methanol masers, one of the key requirements of the current models involves pumping through the first two torsionally excited levels (e.g., Sobolev \& Deguchi 1994; Sobolev et al. 1997a), and maser candidates have been identified in these levels (e.g., the $20.9 \mathrm{GHz} \quad 10_{1} \rightarrow 11_{2} \mathrm{~A}^{+}\left(v_{t}=1\right)$ and the $44.9 \mathrm{GHz}$ $2_{0} \rightarrow 3_{1}$ E $\left(v_{t}=1\right)$ transitions; Sobolev et al. 1997b; Cragg et al. 2005). Despite searches for these torsionally excited lines (e.g., Menten et al. 1986; Voronkov et al. 2002), definitive evidence of maser amplification has not been found (however, both works suggested on the basis of indirect arguments that there might be weak maser emission from these transitions toward $\mathrm{W} 3(\mathrm{OH})$, but no conclusive evidence was available, and neither instance has ever been confirmed). Consequently, 
Table 1

Target Spectral Lines

\begin{tabular}{|c|c|c|c|c|c|c|c|}
\hline Spectral Line & $\begin{array}{l}\text { Rest Freq. } \\
(\mathrm{MHz})\end{array}$ & Epoch & $\begin{array}{c}\text { ATCA } \\
\text { Antennas }\end{array}$ & $\begin{array}{l}V_{\text {coverage }} \\
\left(\mathrm{km} \mathrm{s}^{-1}\right)\end{array}$ & $\begin{array}{c}V_{\text {res. }} \\
\left(\mathrm{km} \mathrm{s}^{-1}\right)\end{array}$ & $\begin{array}{l}\text { Synth. Beam } \\
\left(" \times{ }^{\prime \prime}\right)\end{array}$ & $\begin{array}{c}\text { Rms } \\
\text { Noise (mJy) }\end{array}$ \\
\hline $\mathrm{CH}_{3} \mathrm{OH} 17_{-2} \rightarrow 18_{-3} \mathrm{E}\left(v_{t}=1\right)$ & $6181.128(21)^{4}$ & 190306 & $4,5,6$ & 97 & 0.05 & $3.0 \times 0.9$ & 38 \\
\hline $\mathrm{CH}_{3} \mathrm{OH} 5_{1} \rightarrow 6_{0} \mathrm{~A}^{+}\left(v_{t}=0\right)$ & $6668.5192(8)^{1}$ & 190306 & $4,5,6$ & 89 & 0.05 & $2.8 \times 0.8$ & 34 \\
\hline $\mathrm{CH}_{3} \mathrm{OH} 12_{4} \rightarrow 13_{3} \mathrm{~A}^{+}\left(v_{t}=0\right)$ & $7830.864(50)^{3}$ & 190306 & $4,5,6$ & 95 & 0.05 & $2.3 \times 0.7$ & 32 \\
\hline $\mathrm{CH}_{3} \mathrm{OH} 2_{1} \rightarrow 3_{0} \mathrm{E}\left(v_{t}=0\right)$ & $19967.3961(2)^{1}$ & 190305 & $3,4,5,(6)$ & 961 & 0.47 & $11.4 \times 5.0$ & 20 \\
\hline $\mathrm{CH}_{3} \mathrm{OH} 10_{1} \rightarrow 11_{2} \mathrm{~A}^{+}\left(v_{t}=1\right)$ & $20970.651(50)^{3}$ & 190305 & $3,4,5,(6)$ & 914 & 0.45 & $10.7 \times 4.6$ & 35 \\
\hline $\mathrm{CH}_{3} \mathrm{OH} 7_{-2} \rightarrow 8_{-1} \mathrm{E}\left(v_{t}=0\right)$ & $37703.696(13)^{2}$ & 190307 & $3,4,5,(6)$ & 763 & 0.25 & $6.3 \times 2.5$ & 19 \\
\hline $\mathrm{CH}_{3} \mathrm{OH} 6_{2} \rightarrow 5_{3} \mathrm{~A}^{-}\left(v_{t}=0\right)$ & $38293.292(14)^{2}$ & 190307 & $3,4,5,(6)$ & 500 & 0.24 & $6.2 \times 2.5$ & 22 \\
\hline $\mathrm{CH}_{3} \mathrm{OH} 6_{2} \rightarrow 5_{3} \mathrm{~A}^{+}\left(v_{t}=0\right)$ & $38452.652(14)^{2}$ & 190307 & $3,4,5,(6)$ & 498 & 0.24 & $6.2 \times 2.5$ & 22 \\
\hline $\mathrm{CH}_{3} \mathrm{OH} 7_{0} \rightarrow 6_{1} \mathrm{~A}^{+}$ & $44069.410(10)^{1}$ & 190305 & $3,4,5,(6)$ & 435 & 0.21 & $5.1 \times 2.2$ & 47 \\
\hline $\mathrm{CH}_{3} \mathrm{OH} 2_{0} \rightarrow 3_{1} \mathrm{E}\left(v_{t}=1\right)$ & $44955.807(50)^{3}$ & 190305 & $3,4,5,(6)$ & 426 & 0.21 & $5.0 \times 2.1$ & 48 \\
\hline $\mathrm{CH}_{3} \mathrm{OH} 9_{3} \rightarrow 10_{2} \mathrm{E}\left(v_{t}=0\right)$ & $45843.519(50)^{3}$ & 190305 & $3,4,5,(6)$ & 418 & 0.20 & $4.9 \times 2.2$ & 50 \\
\hline
\end{tabular}

Note. Column two provides the adopted rest frequency with errors in the last digit in parenthesis (including superscript references as follows: 1: Muller et al. (2004); 2: Xu et al. (1997); 3: Tsunekawa et al. (1995); and 4: Pickett et al. (1998)), followed by the observation epoch (YYMMDD), the available ATCA antennas (where (6) indicates that antenna 6 was included in the array but excluded from imaging), observed velocity coverage (approximately centred on $-16 \mathrm{kms}^{-1}$ with respect to the LSR), resultant velocity resolution (after smoothing in the case of the four 6 and $7 \mathrm{GHz}$ transitions), the synthesised beam size and the resultant RMS noise of the observations.

the involvement of torsional levels has remained an open question in the maser pumping theories. In addition to the elusive torsionally excited transitions, many other class II methanol maser lines have been predicted to arise under plausible physical parameter combinations, but are yet to be confirmed by observations (e.g., Sobolev et al. 1997b; Cragg et al. 2005).

The $6.68 \mathrm{GHz}$ methanol maser, G 358.931-0.030, was first detected in the MMB survey with a peak flux density of $10 \mathrm{Jy}$ in early 2006 (Australia Telescope Compact Array (ATCA); follow-up observations of the survey detection were performed on 2006 March 31; Caswell et al. 2010). Parkes 64 m telescope observations of this maser found no accompanying $12.2 \mathrm{GHz}$ emission in either 2008 June or December with $5 \sigma$ detection limits of 0.75 and $0.80 \mathrm{Jy}$, respectively (Breen et al. 2012), but ATCA observations detected a $0.7 \mathrm{Jy} 22 \mathrm{GHz}$ water maser (Titmarsh et al. 2016). Monitoring observations of the $6.68 \mathrm{GHz}$ methanol maser emission have been conducted with the Hitachi $32 \mathrm{~m}$ telescope since 2013 January 2, showing that the source maintained a flux density below $10 \mathrm{Jy}$ until exhibiting an increase in peak flux density from 2019 January 14 (Sugiyama et al. 2019), signifying the start of the maser burst. Previous similar maser flaring events (e.g., in S255IRNIRS3 and NGC 6334; Fujisawa et al. 2015; MacLeod et al. 2018) have been linked to episodic accretion (e.g., Hunter et al. 2017, 2018; Moscadelli et al. 2017) and therefore provide an excellent opportunity to study the mechanisms of high-mass star formation. Using maser monitoring data from a small number of sources (S255IR-NIRS3, G 24.329+0.144, and Cepheus A), Rajabi et al. (2019) suggested that superradiance could play an important role in strong $6.68 \mathrm{GHz}$ methanol maser flaring events. The current flaring event in G 358.931 -0.030 shows rapid variations of numerous maser transitions across a wide range of frequencies and flux densities, providing a challenge for both maser pumping and superradiance theories. Observations of the variability and presence of different maser transitions during a period of bursting activity can therefore provide stringent tests of current theories of the flares in the lines with very high brightness temperatures.
Here we present the results of targeted observations toward G 358.931-0.030 for methanol transitions that have been predicted to produce class II methanol masers, along with one line that is not present in the list of maser candidate transitions, and discuss the implications of a number of new discoveries. The monitoring observations that first discovered the flaring event (Sugiyama et al. 2019) form part of a wider effort by the Maser Monitoring Organisation (M2O), a collaborative international program now monitoring a large number of maser sources and triggering follow-up observations of flaring events (e.g., Burns et al. 2019). The current observations are just one component of a large program following up the maser flare, targeting G 358.931 -0.030 across a broad range of wavelengths, including using very long baseline interferometry (VLBI) observations and singledish monitoring of the maser lines reported here.

\section{Observations and Data Reduction}

A series of spectral line observations targeting the $6.68 \mathrm{GHz}$ methanol maser G 358.931-0.030 (J2000 position: $17^{\mathrm{h}} 43^{\mathrm{m}} 10$. $02-29^{\circ} 51^{\prime} 45$ ". 8 ; Caswell et al. 2010) were undertaken with the ATCA on 2019 March 5, 6, and 7 following successful preliminary Mopra observations conducted on 2019 March 2 and 3 (when emission in the 20.9, 44.9, and $45.8 \mathrm{GHz}$ lines were first discovered, and the $36.2,37.7$, and $44.1 \mathrm{GHz}$ lines were first detected toward this source). In this Letter we limit our results and discussion to the 12 methanol transitions summarized in Table 1.

The ATCA was in the $\mathrm{H} 214$ array configuration, but was in scheduled maintenance that restricted the time and number of antennas (a maximum of four) available for observations (see Table 1 for a summary). A total of four frequency setups were used over three days, comprising a total of 49 spectral zoom windows in the 19.9-25.1 and 41.8-45.9 GHz ranges on 2019 March 5, the 5.0-8.4 GHz range on 2019 March 6, and the 34.4-39.3 GHz range on 2019 March 7. The Compact Array Broadband Backend (CABB; Wilson et al. 2011) was configured in CFB 64M-32k mode on both March 5 and 7, allowing a series of $64 \mathrm{MHz}$ zoom bands, each with 2048 
spectral channels, providing adequate velocity resolution $\left(0.20-0.47 \mathrm{~km} \mathrm{~s}^{-1}\right)$ and coverage $\left(>418 \mathrm{~km} \mathrm{~s}^{-1}\right)$ for millimeter observations. On March 6 CABB was configured in CFB 1M$0.5 \mathrm{k}$ mode, allowing for higher velocity resolution from $1 \mathrm{MHz}$ zooms, each with 2048 spectral channels. Multiple $1 \mathrm{MHz}$ zooms were concatenated in order to provide sufficient velocity coverage $\left(>89 \mathrm{~km} \mathrm{~s}^{-1}\right)$ for the centimeter observations. Each of the frequency setups allowed for an additional two $\times 2 \mathrm{GHz}$ continuum bands, which were centered on 20.7, 24.09, 42.75, and 45.0 on March 5, 6.0 and 7.5 on March 6, and 35.3 and $38.4 \mathrm{GHz}$ on March 7.

At all frequencies, observations of G 358.931-0.030 were interspersed with observations of a nearby phase calibrator at least every 10 minutes; B1714-336 was observed during the 5.0-8.4 GHz observations, and B1741-312 was observed for all other frequencies. All observations were made as a series of cuts over an hour angle range of at least 5 hours, and at all frequencies above $19.9 \mathrm{GHz}$, pointing corrections were made once per hour. Observations of PKS B1253-055 and PKS B1934-638 were carried out for bandpass and primary flux density calibration.

Data were reduced using the MIRIAD software package (Sault et al. 1995). For each observing epoch, a flux model was fit to the broadband PKS B1253-055 continuum data, which was then bootstrapped to PKS B1934-638 for absolute flux density scaling. The resulting flux density model of PKS B1253-055 was then used to determine the bandpass and amplitude scaling of individual zooms. On 2019 March 6, B1741-312 had sufficient parallactic angle coverage to calculate a robust leakage solution. Polarization observations on this day are accurate to within $\sim 0.1 \%$ of Stokes $I$ (Rayner et al. 2000). However, due to insufficient parallactic angle coverage of our phase calibrator on March 5 and 7, PKS B1934-638 was used to calculate the leakage terms and was assumed to have zero polarized flux at all observed frequencies. This is not absolutely known, though comparison between leakage solutions from PKS B1934-638 and B1741-312 on 2019 March 6 differ by $0.3 \%$, giving credence to the assumed polarized flux density of PKS B1934 -638 . Although we are confident in our calibration, we recognize that the small number of antennas, relatively low sensitivity, and imperfect parallactic angle coverage could have introduced small errors. As a result, we have been relatively conservative with our polarization detection limits, only listing detections when they are $>0.5 \%$ of Stokes $I$. The absolute uncertainty in primary flux density calibration is expected to be within $10 \%$.

Given that the ATCA was in an H214 array, data from antenna 6 (located more than $4 \mathrm{~km}$ from all other antennas) was excluded in the imaging of lines at frequencies above $19 \mathrm{GHz}$, leaving only three baselines between 82 and $138 \mathrm{~m}$. Observations between 5.0 and $8.4 \mathrm{GHz}$ were imaged using all three available baselines for that epoch (i.e., including antenna 6), ranging between 138 and $4408 \mathrm{~m}$. Image cubes of each line were created and used to extract absolute positions at the methanol maser peak velocity channel. Caswell (1997) estimated that rms positional uncertainty of ATCA maser observations conducted in this manner to be $\sim 0.4 \operatorname{arcsec}$ (Table 2 shows that the differences in the absolute positions measured for the class II transitions are generally less than $\sim 0.2$ arcsec, even at high frequencies). This was followed by an iteration of phase-only self-calibration using the model created from the clean components in the initial cubes. In some cases, further phase-only self-calibration iterations were required, reflecting the incomplete arrays utilized in these observations. Following self-calibration, Stokes $I, Q, U$, and $V$ images were created and used to make Stokes $I$ as well as circularly and linearly polarized intensity spectra. The velocity resolution of the images (and therefore spectra) are given in Table 1 and are the native resolution of the observations in all cases except for the $6.18,6.68,7.68$ and $7.83 \mathrm{GHz}$ transitions, the data for which have been smoothed to a velocity resolution of $0.05 \mathrm{~km} \mathrm{~s}^{-1}$.

A summary of the methanol transitions, the adopted rest frequencies, epoch of observation, the ATCA antennas available on each day, velocity coverage and resolution (after smoothing in the case of the $6.18,6.68,7.68$, and $7.83 \mathrm{GHz}$ transitions), as well as the resultant synthesized beam sizes and rms noise levels, are given in Table 1 .

\section{Results}

ATCA observations of the 14 methanol transitions (listed in Table 1) toward $6.68 \mathrm{GHz}$ methanol maser G 358.931-0.030 have resulted in the detection of emission in 12 of these transitions, six of which are new class II methanol maser transitions, including the discovery of the first torsionally excited methanol masers. The six new class II methanol maser transitions are those at frequencies of $6.18,7.68,7.83,20.9$, 44.9, and $45.8 \mathrm{GHz}$ (indicated in bold in Table 2). Here we also report the first detection of $36.2 \mathrm{GHz}$ class I methanol maser emission and $37.7 \mathrm{GHz}$ class II maser emission toward this source. We made independent detections of the 19.9, 23.1, and $44.1 \mathrm{GHz}$ transitions but subsequently found that these had been discovered slightly earlier than our observations (e.g., K.-T. Kim et al. 2019, in preparation; G. MacLeod et al. 2019, in preparation; Volvach et al. 2019).

The properties of the detected maser lines are provided in Table 2 , including the fitted position at the maser peak velocity, flux density, and velocity information, along with percentage of linear and circular polarization. As shown, we find only three examples of circularly polarized emission, the most significant ( $\sim 1.5 \%$ of the Stokes $I$ flux density) in the $45.8 \mathrm{GHz}$ transition, but find significant linear polarization $(>0.5 \%$ of the Stokes $I$ flux density) to be relatively common.

Spectra of each of the detected lines are shown in Figure 1 and include both Stokes $I$ and the linearly polarized emission (scaled by a factor of 10). Despite being co-spatial, some variation in the velocity of the detected maser features can be seen across the transitions. These can all be accounted for by the uncertainty in the adopted rest frequencies, which are shown in Tables 1 and 2. Comparison between the velocities shows that the largest deviations from the $6.68 \mathrm{GHz}$ maser velocity is in the $6.18,7.68$, and $7.83 \mathrm{GHz}$ lines. We suggest that refinements of the respective rest frequencies to 6181.146, 7682.246 , and $7830.848 \mathrm{MHz}$ would result in much closer velocity correspondence between all of the class II methanol maser transitions.

The derived positions listed in Table 2 confirm that all of the detected class II methanol maser lines are co-spatial with the $6.68 \mathrm{GHz}$ methanol maser, and show a slight $\left(\sim 1^{\prime \prime}\right)$ variation from the published MMB position (Caswell et al. 2010). The MMB position for the $6.68 \mathrm{GHz}$ methanol maser was measured when the peak flux density was $6 \mathrm{Jy}$, a factor of $>100$ lower than for the current observations, which, combined with the inferior receiver system available at the time of the observations, can likely account for the discrepancy. Imaging of the 
Table 2

Properties of the Methanol Masers Detected Toward G 358.931-0.030

\begin{tabular}{|c|c|c|c|c|c|c|c|c|c|c|}
\hline Spectral Line & $\begin{array}{l}\text { R.A. (2000) } \\
\quad\left(\mathrm{h} \mathrm{m} \mathrm{s}^{2}\right)\end{array}$ & $\begin{array}{l}\text { Decl. (2000) } \\
\quad\left({ }^{\prime},{ }^{\prime \prime}\right)\end{array}$ & \multicolumn{3}{|c|}{$\left(\mathrm{km} \mathrm{s}^{-1}\right)$} & $V_{\text {Uncert. }}$ & $\begin{array}{c}S_{\text {peak }} \\
(\mathrm{Jy})\end{array}$ & $\begin{array}{c}S_{\text {int }} \\
\left(\mathrm{Jy} \mathrm{km} \mathrm{s}^{-1}\right)\end{array}$ & $\begin{array}{l}\text { Linear } \\
\text { pol. } \%\end{array}$ & $\begin{array}{c}\text { Circular } \\
\text { pol. } \%\end{array}$ \\
\hline $6.18 \mathrm{GHz}$ & 174310.10 & -295145.6 & -18.8 & -15.5 & -16.2 & $(1.02)$ & 290 & 340 & 7.0 & $<0.5$ \\
\hline $6.68 \mathrm{GHz}$ & $1743 \quad 10.10$ & -295145.5 & -19.6 & -12.7 & -17.2 & $(0.04)$ & 981 & 1110 & 7.5 & 0.5 \\
\hline $7.83 \mathrm{GHz}$ & 174310.10 & -295145.6 & -19.3 & -12.6 & -16.6 & $(1.92)$ & 571 & 711 & 3.5 & $<0.5$ \\
\hline $19.9 \mathrm{GHz}$ & $1743 \quad 10.14$ & -295145.9 & -18.1 & -14.8 & -17.6 & (0.003) & 0.3 & 0.7 & $<0.5$ & $<0.5$ \\
\hline $20.9 \mathrm{GHz}$ & 174310.12 & -295145.8 & -33.5 & -0.7 & -15.6 & $(0.71)$ & 978 & 1896 & 7.0 & $<0.5$ \\
\hline $37.7 \mathrm{GHz}$ & 174310.10 & -295145.3 & -22.3 & -10.8 & -17.3 & $(0.10)$ & 249 & 496 & 3.5 & 0.5 \\
\hline $44.1 \mathrm{GHz}$ & 174309.92 & -295146.0 & -22.4 & -16.5 & -21.1 & $(0.07)$ & 4.5 & 4.4 & $<0.5$ & $<0.5$ \\
\hline $44.9 \mathrm{GHz}$ & $1743 \quad 10.11$ & -295145.4 & -22.1 & -10.1 & -15.4 & $(0.33)$ & 508 & 536 & 2.5 & $<0.5$ \\
\hline $45.8 \mathrm{GHz}$ & 174310.10 & -295145.4 & -22.4 & -9.8 & -15.4 & $(0.33)$ & 414 & 555 & 7.0 & 1.5 \\
\hline
\end{tabular}

Note. The measured positions are provided in columns 2 and 3, and are followed by the minimum, maximum and peak velocity, uncertainty in velocity (based on the uncertainty in the respective rest frequencies), the peak and integrated flux densities, as well as the maximum linear and circular polarisation percentage. The newly discovered class II maser transitions are indicated in bold font.

$6.68 \mathrm{GHz}$ transition being undertaken with other interferometers will provide further independent estimates of the absolute position in the near future. The largest deviation in our measured class II positions is in the weakest transition at $19.9 \mathrm{GHz}$, which is $\sim 0$ ". 5 from our measured location of the $6.68 \mathrm{GHz}$ maser. The location of the peak class I methanol maser emission in both the 36.2 and $44.1 \mathrm{GHz}$ transitions is offset by $\sim 1$ ". 5 from the class II maser position, and the precise correspondence between the 36.2 and $44.1 \mathrm{GHz}$ lines indicate that this offset is genuine.

\section{Discussion}

\subsection{Newly Discovered $\mathrm{v}_{\mathrm{t}}=1$ Methanol Masers at 6.18, 20.9, and $44.9 \mathrm{GHz}$}

The clear detection of torsionally excited methanol maser lines resolves a significant outstanding uncertainty with current maser models, unambiguously demonstrating that pumping through the torsionally excited levels occurs, as required by the models, for the strong, commonly observed transitions. We detected three torsionally excited methanol masers with rest frequencies near $6.18,20.9$, and $44.9 \mathrm{GHz}$; the latter two were included in maser models and have been listed as maser candidates under some model conditions (e.g., Cragg et al. 2005). However, our current data shows much higher intensities than the models predict, and therefore the current observations offer substantial new information for methanol maser pumping models.

Previous observations of the 20.9 and $44.9 \mathrm{GHz}$ transitions have detected a handful of emission sites: Menten et al. (1986) targeted nine sources for the $20.9 \mathrm{GHz}$ transition, and detected emission toward $\mathrm{W} 3(\mathrm{OH})$, Orion $\mathrm{KL}$ and $\mathrm{W} 51 \mathrm{E}$, while Voronkov et al. (2002) observed six strong class II methanol maser sites and Orion $\mathrm{KL}$ at $44.9 \mathrm{GHz}$, reporting detections toward Orion $\mathrm{KL}, \mathrm{W} 3(\mathrm{OH})$, and NGC 6334F. In both cases, $\mathrm{W} 3(\mathrm{OH})$ showed some evidence of possible weak maser emission, although this has never been confirmed. A search for the $44.9 \mathrm{GHz}$ transition toward a sample of 272 sources with excess $4.5 \mu \mathrm{m}$ emission in the Spitzer GLIMPSE survey has been conducted with the Mopra telescope, and even though more than $40 \%$ of these sources have nearby $6.68 \mathrm{GHz}$ methanol masers, no emission was detected to a limit of
2 Jy (S. P. Ellingsen et al. 2019, in preparation), demonstrating that strong emission in this transition is very rare.

The $6.18 \mathrm{GHz}$ methanol transition was not included in the published lists of class II methanol maser candidates (see, e.g., Cragg et al. 2005 for the most comprehensive list), and the only previously reported search toward the rich class II methanol maser site, G 345.01+1.79, found no emission above a $3 \sigma$ detection limit of $0.15 \mathrm{Jy}$ (Chipman et al. 2016). The detection of such a highly excited transition suggests that we are observing a previously unidentified pumping regime for the class II methanol masers. Modeling of this line requires further development of the methanol molecule scheme of levels and transitions, beyond the data available at present.

\subsection{Newly Discovered Class II $\mathrm{v}_{\mathrm{t}}=0$ Methanol Maser Transitions at 7.68, 7.83, and $45.8 \mathrm{GHz}$}

All three of the newly discovered $v_{t}=0$ class II methanol masers (at 7.68, 7.83, and $45.8 \mathrm{GHz}$ ) were predicted to exhibit maser emission under some conditions (Cragg et al. 2005), but no maser emission has been reported in any sources. The only published search for any of these transitions was by Chipman et al. (2016), who targeted four of the most prominent sites of class II methanol maser emission (G 339.88-1.26, G 345.01 +1.79 , NGC 6334F, and W48) in the 7.68 and $7.83 \mathrm{GHz}$ lines, finding no emission above a $3 \sigma$ detection limit of $0.11 \mathrm{Jy}$.

\subsection{The Known Class II Methanol Maser Transitions at 6.68,} 19.9, 23.1, 37.7, 38.3, and $38.5 \mathrm{GHz}$

Our observations detected a strong $37.7 \mathrm{GHz}$ methanol maser at the location of G 358.931-0.030, but found no accompanying emission in the 38.3 and $38.5 \mathrm{GHz}$ transitions to $3 \sigma$ detection limits of $66 \mathrm{mJy}$. Recent sensitive, highresolution observations of the $37.7,38.3$, and $38.5 \mathrm{GHz}$ methanol maser lines toward 11 known $37.7 \mathrm{GHz}$ sources found that all $37.7 \mathrm{GHz}$ methanol masers stronger than $16 \mathrm{Jy}$ had accompanying emission in both the 38.3 and $38.5 \mathrm{GHz}$ transitions, with ratios ranging from 300:1 and 400:1 (in G 339.8841.259) to $0.31: 1$ and $0.26: 1$ (in NGC 6334F) (Ellingsen et al. 2018). The lower limit of the $37.3 \mathrm{GHz}$ to 38.3 and $38.5 \mathrm{GHz}$ flux density ratio in $\mathrm{G} 358.931-0.030$ is 

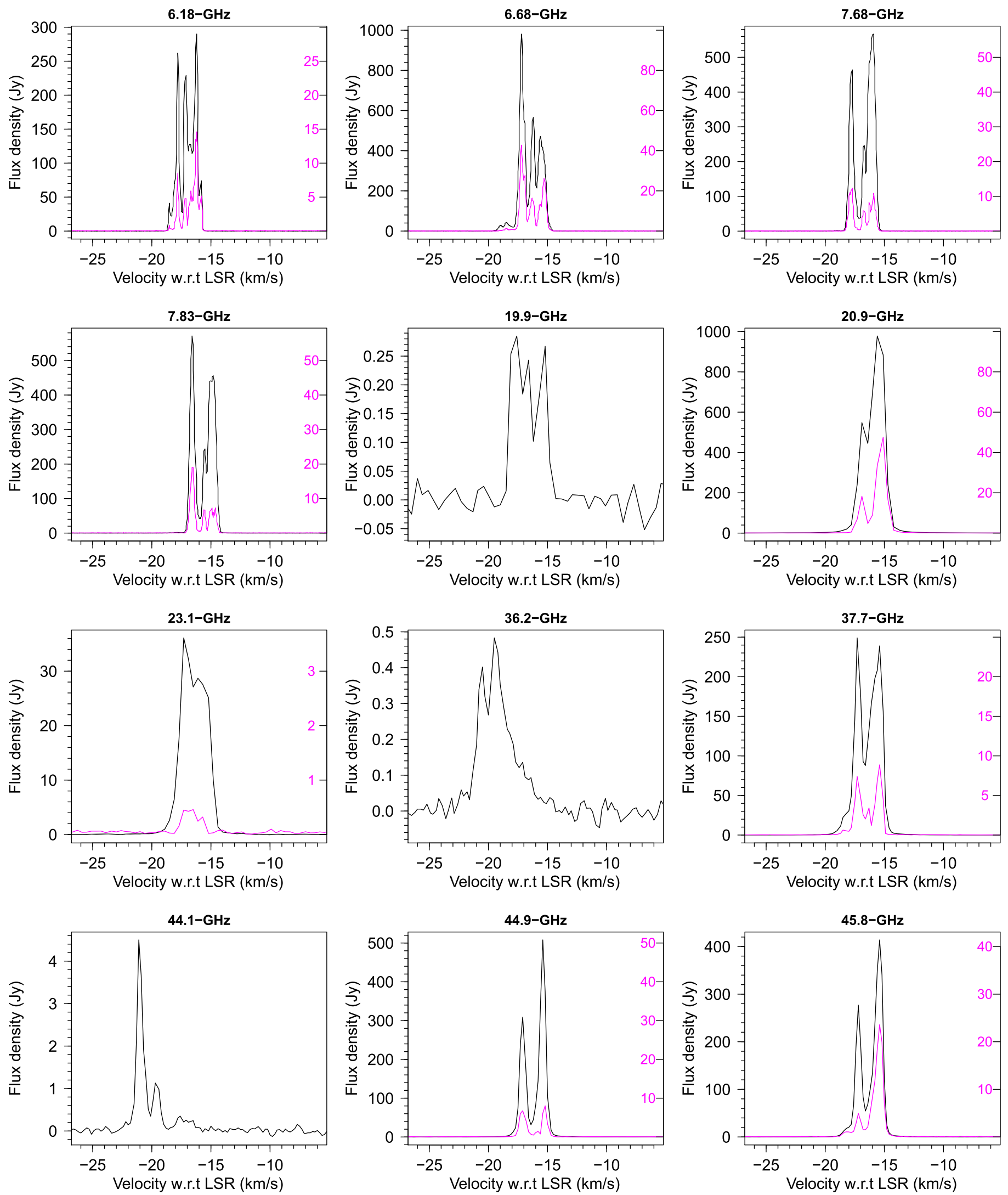

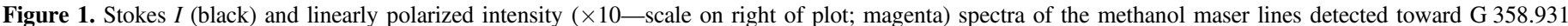
-0.030 . Note that the $19.9,36.2$, and $44.1 \mathrm{GHz}$ transitions have no detected linearly polarized emission.

$\sim 377: 1$, indicating that the conditions in this source are at the extreme end of known sources exhibiting $37.7 \mathrm{GHz}$ maser emission.
The $6.68 \mathrm{GHz}$ methanol maser was discovered in the MMB survey with a peak flux density of $10 \mathrm{Jy}$ (Caswell et al. 2010) and was reported by Sugiyama et al. (2019) to be undergoing a 
period of bursting activity on 2019 January 21 (when it had a peak flux density of $19 \mathrm{Jy}$, quickly rising to $99 \mathrm{Jy}$ by January 26). Our observations, 37 days later, now measure a peak flux density of $981 \mathrm{Jy}$. The report of flaring in the $6.68 \mathrm{GHz}$ maser emission triggered follow-up observations of a number of different known class II methanol maser transitions with the 19.9 and $23.1 \mathrm{GHz}$ transitions having been detected by other telescopes prior to our observations by the $\mathrm{M} 2 \mathrm{O}$ collaboration. The current observations confirm that both of these transitions arise from the same region as the $6.68 \mathrm{GHz}$ methanol masers.

\subsection{Class I Methanol Masers at 36.2 and $44.1 \mathrm{GHz}$}

This is the first reported detection of class I methanol maser emission in the $36.2 \mathrm{GHz}$ transition toward G 358.931-0.030. We also report on our observations of the $44.1 \mathrm{GHz}$ transition, which also independently detected by other members of the M2O collaboration (Kim et al. 2019, in preparation). Although class I methanol masers in the 36.2 and $44.1 \mathrm{GHz}$ lines are relatively common in the vicinity of young high-mass stars harboring $6.68 \mathrm{GHz}$ masers (e.g., Voronkov et al. 2014), observations of these lines only cover a small fraction of the Galaxy. Without pre-burst observations we are unable to conclude whether or not these masers have appeared due to the ongoing flaring event, or how they have been effected.

\subsection{Class II Methanol Maser Polarization}

We detect modest levels of linearly polarized emission in the $6.18,6.68,7.68 .7 .83,20.9,23.1,37.7,44.9$, and $45.8 \mathrm{GHz}$ transitions, with maximum polarized components in the range from $1.5 \%$ to $7.5 \%$ (listed in Table 2). We additionally detect low levels of circular polarization in the $6.68,37.7$, and $45.8 \mathrm{GHz}$ transitions in the $0.5 \%-1.5 \%$ range. The levels of linear polarization that we detect are similar to those found in $6.68 \mathrm{GHz}$ class II methanol masers by previous studies (a few to $\sim 10 \%$, e.g., Stack \& Ellingsen 2011; Surcis et al. 2019), who similarly found that circularly polarized components are scarce. Given the limitations of the current observations, further polarization observations will be needed to determine a more thorough picture.

\subsection{Class II Methanol Maser Flaring Events}

Caswell et al. (1995) observed more than $2006.68 \mathrm{GHz}$ methanol masers at multiple epochs over a period of approximately 18 months and concluded that while variability of class II methanol masers was common, it was rarely large in amplitude and, furthermore, that higher intensity sources were generally less variable. While a comparison of the spectra of a sample of strong $6.68 \mathrm{GHz}$ methanol masers over a $20 \mathrm{yr}$ time baseline was used to estimate that individual spectral components have a typical lifetime of around $150 \mathrm{yr}$ (Ellingsen 2007). These and other studies of the variability of class II methanol masers resulted in the general consensus that the large-amplitude, rapid variability that is relatively common in $22 \mathrm{GHz}$ water masers (e.g., Felli et al. 2007) is either absent, or rare in class II methanol masers. However, an increase in the active monitoring of $6.68 \mathrm{GHz}$ methanol masers has detected several examples of extreme flaring in class II methanol masers (e.g., Fujisawa et al. 2015; MacLeod et al. 2018). At the time of writing, the flare in the class II maser emission toward G 358.931-0.030 is ongoing, and the timely sharing of results and collaboration within the maser community has resulted in an unprecedented wealth of data covering many different molecular transitions. Previous class II methanol flares have generally only been observed in one or both of the 6.68 and $12.2 \mathrm{GHz}$ methanol transitions. The flare in G 358.931-0.030 has demonstrated that where extreme and rapid variability is seen in the $6.68 \mathrm{GHz}$ transition, it may be accompanied by emission in other much rarer class II methanol transitions. We encourage further multi-wavelength studies of this source to complement the molecular studies and aid in their interpretation.

Flaring masers offer a particularly good opportunity to test maser pumping theories and detect new and uncommon masers due to the relatively rapid changes in the radiation field. The pumping cycles of methanol masers are significant and time is required to achieve equilibrium in the maser level populations (e.g., Sobolev \& Deguchi 1994). This means that in the case where the radiation field is changing rapidly, we are dealing with masers in the non-stationary regime where some new and previously unpredicted maser transitions, like the one at $6.18 \mathrm{GHz}$, can occur. These effects are not yet studied and their significance will be elucidated by theoretical modeling based on results of an ongoing monitoring effort of the detections reported here.

The refinement of the maser pumping models will allow us to use sensitive observations covering multiple maser transitions to infer physical conditions in the masing gas. In particular, the less-common transitions are generally inverted over a narrower range of physical conditions in pumping models and, where emission is shown to be co-spatial, the relative intensity of the different transitions will significantly constrain the possible physical conditions. A number of such studies have been undertaken toward high-mass star formation regions that exhibit a large number of class II methanol maser transitions (e.g., Cragg et al. 2001, 2005; Sutton et al. 2001). However, they combined data collected over a period of many years using multiple telescopes (generally single-dish instruments), and so interpretation was limited by uncertainty due to potential variability and a lack of information as to the degree to which the emission from the different transitions was cospatial. Our observations show that the 10 class II methanol maser transitions that we have detected are co-spatial to around 0 ". 2 (which is within the systematic positional uncertainty of the observations). The intense emission in such a large number of different class II methanol maser transitions presents a unique opportunity to undertake milliarcsecond-scale observations (using VLBI) and determine the degree to which they are co-spatial on milliarcsecond scales. Where that is found to be the case, detailed maser modeling can potentially infer the physical conditions at unprecedented resolution for high-mass star formation regions - scales of a few au for individual maser spots and variations in the conditions on scales of tens to hundreds of au (the size of the maser clusters).

\section{Summary}

We have discovered six new class II methanol maser transitions toward G 358.931-0.030, a $6.68 \mathrm{GHz}$ methanol maser that was recently reported to be undergoing a period of flaring. These discoveries include three transitions in the torsionally excited $\left(v_{t}=1\right)$ state, the $6.18 \mathrm{GHz} 17_{-2} \rightarrow 18_{-3}$ $\mathrm{E}\left(v_{t}=1\right), 20.9 \mathrm{GHz} 10_{1} \rightarrow 11_{2} \mathrm{~A}^{+}\left(v_{t}=1\right)$, and $44.9 \mathrm{GHz}$ $2_{0} \rightarrow 3_{1}$ E $\quad\left(v_{t}=1\right)$ transitions, and comprise the first torsionally maser lines ever definitively detected. The detection of these lines validates predictions of methanol maser pumping 
models, but have unexpectedly high flux densities and so provide significant new information to refine current methanol maser pumping theories.

In addition to the torsionally excited lines, we have discovered three new class II methanol masers in the $7.86 \mathrm{GHz}$ $12_{4} \rightarrow 13_{3} \mathrm{~A}^{-}\left(v_{t}=0\right), 7.83 \mathrm{GHz} 12_{4} \rightarrow 13_{3} \mathrm{~A}^{+}\left(v_{t}=0\right)$, and $5.8 \mathrm{GHz} 9_{3} \rightarrow 10_{2} \mathrm{E}\left(v_{t}=0\right)$ transitions, and the first detections of the $4_{-1} \rightarrow 3_{0}$ E $36.2 \mathrm{GHz}$ and $7_{-2} \rightarrow 8_{-1}$ $\mathrm{E}\left(v_{t}=0\right) 37.7 \mathrm{GHz}$. We present upper limits on the $6_{2} \rightarrow 5_{3} \mathrm{~A}^{-}$and $\mathrm{A}^{+}$transitions at 38.3 and $38.5 \mathrm{GHz}$ and show that the detected $37.7 \mathrm{GHz}$ maser has an usually high flux density to exhibit no accompanying emission in the 38.3 and $38.5 \mathrm{GHz}$ transitions.

We suggest refinements of the $6.18,7.68$, and $7.83 \mathrm{GHz}$ rest frequencies to $6181.146,7682.246$, and $7830.848 \mathrm{MHz}$, respectively, in order to achieve velocity correspondence with the $6.68 \mathrm{GHz}$ methanol maser emission.

The relative total, linearly, and circularly polarized intensities of the large number of detected maser lines will be used in combination with the plethora of multi-wavelength observations (including infrared, maser VLBI, and maser monitoring observations) to both inform maser pumping schemes as well as to infer the usual physical conditions associated with the flaring event.

The Australia Telescope Compact Array and Mopra radio telescope are part of the Australia Telescope National Facility. This research has made use of NASA's Astrophysics Data System Abstract Service. S.P.E. acknowledges the support of ARC Discovery Project (project number DP180101061). A.M.S. was supported by the Russian Science Foundation (grant 18-1200193).

\section{ORCID iDs}

S. L. Breen (1) https://orcid.org/0000-0002-4047-0002

A. M. Sobolev 10 https://orcid.org/0000-0001-7575-5254

J. F. Kaczmarek (1) https://orcid.org/0000-0003-4810-7803

S. P. Ellingsen (1) https://orcid.org/0000-0002-1363-5457

T. P. McCarthy (1) https://orcid.org/0000-0001-9525-7981

M. A. Voronkov (i) https://orcid.org/0000-0002-4931-4612

\section{References}

Batrla, W., Matthews, H. E., Menten, K. M., \& Walmsley, C. M. 1987, Natur, 326,49

Breen, S. L., Contreras, Y., Dawson, J. R., et al. 2019, MNRAS, 484, 5072 Breen, S. L., Ellingsen, S. P., Caswell, J. L., et al. 2012, MNRAS, 421, 1703 Breen, S. L., Ellingsen, S. P., Caswell, J. L., et al. 2016, MNRAS, 459, 4066 Breen, S. L., Ellingsen, S. P., Contreras, Y., et al. 2013, MNRAS, 435, 524 Breen, S. L., Fuller, G. A., Caswell, J. L., et al. 2015, MNRAS, 450, 4109 Burns, R. A., Orosz, G., Bayandina, O., et al. 2019, MNRAS, submitted Caswell, J. L. 1997, MNRAS, 289, 203

Caswell, J. L., Fuller, G. A., Green, J. A., et al. 2010, MNRAS, 404, 1029

Caswell, J. L., Vaile, R. A., Ellingsen, S. P., Whiteoak, J. B., \& Norris, R. P. 1995, MNRAS, 272, 96

Caswell, J. L., Yi, J., Booth, R. S., \& Cragg, D. M. 2000, MNRAS, 313, 599

Chen, X., Ellingsen, S. P., Shen, Z.-Q., Titmarsh, A., \& Gan, C.-G. 2011, ApJS, 196, 9
Chipman, A., Ellingsen, S. P., Sobolev, A. M., \& Cragg, D. M. 2016, PASA, 33, e056

Cragg, D. M., Sobolev, A. M., Caswell, J. L., Ellingsen, S. P., \& Godfrey, P. D. 2004, MNRAS, 351, 1327

Cragg, D. M., Sobolev, A. M., Ellingsen, S. P., et al. 2001, MNRAS, 323, 939

Cragg, D. M., Sobolev, A. M., \& Godfrey, P. D. 2005, MNRAS, 360, 533

Cyganowski, C. J., Brogan, C. L., Hunter, T. R., \& Churchwell, E. 2009, ApJ, 702,1615

Ellingsen, S. P. 2005, MNRAS, 359, 1498

Ellingsen, S. P. 2007, MNRAS, 377, 571

Ellingsen, S. P., Breen, S. L., Sobolev, A. M., et al. 2011, ApJ, 742, 109

Ellingsen, S. P., Cragg, D. M., Lovell, J. E. J., et al. 2004, MNRAS, 354, 401

Ellingsen, S. P., Cragg, D. M., Minier, V., Muller, E., \& Godfrey, P. D. 2003, MNRAS, 344, 73

Ellingsen, S. P., Sobolev, A. M., Cragg, D. M., \& Godfrey, P. D. 2012, ApJL, 759, L5

Ellingsen, S. P., Voronkov, M. A., Breen, S. L., Caswell, J. L., \& Sobolev, A. M. 2018, MNRAS, 480, 4851

Felli, M., Brand, J., Cesaroni, R., et al. 2007, A\&A, 476, 373

Fujisawa, K., Yonekura, Y., Sugiyama, K., et al. 2015, ATel, 8286, 1

Green, J. A., Caswell, J. L., Fuller, G. A., et al. 2009, MNRAS, 392, 783

Hunter, T. R., Brogan, C. L., MacLeod, G., et al. 2017, ApJL, 837, L29

Hunter, T. R., Brogan, C. L., MacLeod, G. C., et al. 2018, ApJ, 854, 170

Jordan, C. H., Walsh, A. J., Breen, S. L., et al. 2017, MNRAS, 471, 3915

Jordan, C. H., Walsh, A. J., Lowe, V., et al. 2015, MNRAS, 448, 2344

Kurtz, S., Hofner, P., \& Álvarez, C. V. 2004, ApJS, 155, 149

Leurini, S., Menten, K. M., \& Walmsley, C. M. 2016, A\&A, 592, A31

MacLeod, G. C., Smits, D. P., Goedhart, S., et al. 2018, MNRAS, 478, 1077

Menten, K. M. 1991, ApJL, 380, L75

Menten, K. M., Walmsley, C. M., Henkel, C., et al. 1986, A\&A, 169, 271

Minier, V., Ellingsen, S. P., Norris, R. P., \& Booth, R. S. 2003, A\&A, 403, 1095

Moscadelli, L., Sanna, A., Goddi, C., et al. 2017, A\&A, 600, L8

Müller, H. S. P., Menten, K. M., \& Mäder, H. 2004, A\&A, 428, 1019

Pickett, H. M., Poynter, R. L., Cohen, E. A., et al. 1998, JQSRT, 60, 883

Rajabi, F., Houde, M., Bartkiewicz, A., et al. 2019, MNRAS, 484, 1590

Rayner, D. P., Norris, R. P., \& Sault, R. J. 2000, MNRAS, 319, 484

Sault, R. J., Teuben, P. J., \& Wright, M. C. H. 1995, in ASP Conf. Ser. 77, Astronomical Data Analysis Software and Systems IV, ed. R. A. Shaw, H. E. Payne, \& J. J. E. Hayes (San Francisco, CA: ASP), 433

Sobolev, A. M., Cragg, D. M., \& Godfrey, P. D. 1997a, A\&A, 324, 211

Sobolev, A. M., Cragg, D. M., \& Godfrey, P. D. 1997b, MNRAS, 288, L39

Sobolev, A. M., \& Deguchi, S. 1994, A\&A, 291, 569

Sobolev, A. M., \& Parfenov, S. Y. 2018, in IAU Symp. 336, Astrophysical Masers: Unlocking the Mysteries of the Universe, ed. A. Tarchi, M. J. Reid, \& P. Castangia (Cambridge: Cambridge Univ. Press), 57

Stack, P. D., \& Ellingsen, S. P. 2011, PASA, 28, 338

Sugiyama, K., Saito, Y., Yonekura, Y., \& Momose, M. 2019, ATel, 12446, 1

Surcis, G., Vlemmings, W. H. T., van Langevelde, H. J., Hutawarakorn Kramer, B., \& Bartkiewicz, A. 2019, A\&A, 623, A130

Sutton, E. C., Sobolev, A. M., Ellingsen, S. P., et al. 2001, ApJ, 554, 173

Titmarsh, A. M., Ellingsen, S. P., Breen, S. L., Caswell, J. L., \& Voronkov, M. A. 2016, MNRAS, 459, 157

Tsunekawa, S., Ukai, T., Toyama, A., \& Takagi, K. 1995, Report for the Grantin-aid for Scientific Research on Priority Areas (Interstellar Matter, 1991-1994) of the Ministry of Education, Science and Culture, Japan. Technical Report, (Toyama: Toyama Univ.)

Volvach, A. E., Volvach, L. N., \& Larionov, M. G. 2019, AstL, submitted

Voronkov, M. A., Austin, M. C., \& Sobolev, A. M. 2002, A\&A, 387, 310

Voronkov, M. A., Caswell, J. L., Ellingsen, S. P., Green, J. A., \& Breen, S. L. 2014, MNRAS, 439, 2584

Wilson, W. E., Ferris, R. H., Axtens, P., et al. 2011, MNRAS, 416, 832

Xu, L.-H., \& Lovas, F. J. 1997, JPCRD, 26, 17

Xu, Y., Li, J. J., Hachisuka, K., et al. 2008, A\&A, 485, 729

Yusef-Zadeh, F., Cotton, W., Viti, S., Wardle, M., \& Royster, M. 2013, ApJL, 764, L19

Zinchenko, I., Liu, S.-Y., Su, Y.-N., \& Sobolev, A. M. 2017, A\&A, 606, L6 